TRANSACTIONS OF THE

AMERICAN MATHEMATICAL SOCIETY

Volume 281. Number 2. February 1984

\title{
ON BLOCK'S CONDITION FOR SIMPLE PERIODIC ORBITS OF FUNCTIONS ON AN INTERVAL
}

BY

CHUNG-WU HO

\begin{abstract}
Recently, L. Block has shown that for any mapping $f$ of an interval, whether $f$ has a periodic point whose period contains an odd factor greater than 1 depends entirely on the periodic orbits of $f$ whose periods are powers of 2 . In this paper the author shows that Block's result is a special case of a more general phenomenon.
\end{abstract}

I. Introduction. Let $I$ be a closed bounded interval of the real line, and $C^{0}(I, I)$ the set of all the continuous functions from $I$ into $I$. Given an element $f \in C^{0}(I, I)$, we shall call a point $p \in I$ a periodic point of period $n$ for $f$ if $f^{n}(p)=p$ but $f^{i}(p) \neq p$ for $0<i<n$. A. N. Sharkovskii proved a beautiful theorem about the periodic points of the functions in $C^{0}(I, I)$. Arrange the positive integers in the following sequence:

$$
3,5,7, \ldots, 3 \cdot 2,5 \cdot 2,7 \cdot 2, \ldots, 3 \cdot 4,5 \cdot 4, \ldots, 3 \cdot 8, \ldots, \ldots, 2^{3}, 2^{2}, 2,1 .
$$

Let $n$ be any positive integer. Sharkovskii proved that if $f \in C^{0}(I, I)$ has a periodic point of period $n$, it must also have periodic points for each period following $n$ in the above sequence ([6,7]; see also [2 or 4]). Recently, L. Block has shown in [1] that for any $f \in C^{0}(I, I)$, whether $f$ has a periodic point whose period contains an odd factor greater than 1 depends entirely on the periodic orbits of $f$ whose periods are powers of 2. In this paper, we shall show that Block's result in [1] is a special case of a more general phenomenon. Let us first describe Block's result in more precise terms. Given $f \in C^{0}(I, I)$, we shall let $S_{k}(f)$ be the set of all the periodic points of $f$ of a period $m \cdot 2^{k}$ for some odd integer $m>3$. Also let $S_{\infty}(f)$ be the set of all the periodic points of $f$ whose period is a power of 2. Block first studied the elements of $S_{\infty}$ for a given function $f$. He called a periodic orbit of period a power of 2 a simple orbit if the orbit satisfies certain special conditions (to be described later in §II). He then showed that for any $f \in C^{0}(I, I)$, there exists a point in $S_{k}(f)$ for some nonnegative integer $k$ if and only if there exists a point in $S_{\infty}(f)$ whose orbit is not a simple orbit [1, Theorem A]. In this paper we shall extend Block's condition to orbits whose order is not a power of 2. This condition will simply be called Block's condition (see Definition 2.3). Our main result in this paper can now be stated as follows.

Received by the editors July 8, 1982 and, in revised form, February 28, 1983.

1980 Mathematics Subject Classification. Primary 54H20, 26A18; Secondary 58F20.

'Partially supported by Southern Illinois University at Edwardsville Research Scholar Award. 
THEOREM I. Let any $f \in C^{0}(I, I)$ be given.

(1) If there exists a point in the set $S_{k}(f)$ for any nonnegative integer $k$, then for each $r>k(r=$ positive integer or $\infty)$, there exist infinitely many points in $S_{r}(f)$ such that the orbit of each such point violates Block's condition.

(2) If there exists a point $p$ in the set $S_{r}(f)$ for some $r(r=$ positive integer or $\infty)$ such that the orbit of $p$ violates Block's condition, then there exists a nonnegative integer $k<r$ such that the set $S_{k}(f)$ is nonempty.

Our Theorem I consists of two parts. Part (1) is an immediate consequence of the following theorem, which will be proved in \$III of this paper.

THEOREM II. If $f$ has a periodic point of period $m \cdot 2^{k}$ for some nonnegative integer $k$ and some odd integer $m>1$, then for each of the following integers $N$ :

(1) $N=(m+n) \cdot 2^{k+1}$ for any positive even integer $n$,

(2) $N=(3+n) \cdot 2^{k+2}$ for any positive even integer $n$,

(3) $N=s \cdot 2^{r}$ for any integer $r>k+2$, and any positive integer $s>1$,

$f$ has a periodic point of period $N$, whose orbit violates Block's condition.

Part (2) of our Theorem I can be proved by using [5, Lemma 1.6] or the proof of [2, Lemma 2.1]. The remainder of this paper will be devoted solely to the proof of Theorem II. We shall first collect some necessary preliminaries in §II. The theorem itself will be proved in §III of this paper.

II. Preliminaries. In this section, we shall develop some necessary machinery for a proof of our Theorem II. Let $I_{1}, I_{2}, \ldots, I_{k}$ be a set of closed subintervals of the interval $I$ such that the interiors of $I_{1}, I_{2}, \ldots, I_{k}$ are pairwise disjoint. Let an $f \in C^{0}(I, I)$ be given. We may define a digraph (i.e., a directed graph) associated with the intervals $I_{1}, I_{2}, \ldots, I_{k}$ and $f$ as follows. The graph has a vertex corresponding to each interval $I_{i}$. These vertices will again be denoted by $I_{1}, I_{2}, \ldots, I_{k}$. A directed arc is drawn from the vertex $I_{i}$ to the vertex $I_{j}$ if and only if $f\left(I_{i}\right) \supset I_{j}$. A cycle in this digraph is a sequence of directed arcs such that the end point of each arc coincides with the initial point of the next arc, and the end point of the last arc coincides with the initial point of the first arc. The reason for introducing such a digraph is that we can use it to predict the existence of periodic points of a function by means of the following proposition, which can be proved easily (cf. [8, Theorem A]).

Proposition 2.1. Let $I_{1}, I_{2}, \ldots, I_{k}$ be closed subintervals of the interval $I$, and let $f \in C^{0}(I, I)$ be given. If the associated digraph contains a cycle $J_{1} J_{2} \cdots J_{n}$ for some vertices $J_{1}, J_{2}, \ldots, J_{n}$ of the digraph, then there exists a point $p \in J_{1}$ such that $f^{i}(p) \in J_{i+1}$ for each $i=1,2, \ldots, n-1$, and $f^{n}(p)=p$.

A periodic point $p$ of period $n$ for a function $f \in C^{0}(I, I)$ will be called a minimum periodic point of $f$ if $f$ does not have any periodic point of a period preceding $n$ in the Sharkovskii sequence. P. Štefan proved the following proposition for minimum periodic orbits of an odd order (see [7, Theorem 2]). 
Proposition 2.2. If $P$ is a minimum periodic orbit of an odd order $m$ for some function $f \in C^{0}(I, I)$, then there is a point $p_{1} \in P$ such that

$$
p_{m-1}<p_{m-3}<\cdots<p_{2}<p_{1}<p_{3}<\cdots<p_{m-2}<p_{m}
$$

or

$$
p_{m}<p_{m-2}<\cdots<p_{3}<p_{1}<p_{2}<\cdots<p_{m-3}<p_{m-1}
$$

where for each $i$ with $1<i<m-1, f\left(p_{i}\right)=p_{i+1}$, and $f\left(p_{m}\right)=p_{1}$.

On the other hand, a minimum orbit of an even order must satisfy a different set of conditions which we now describe. Let $P$ be an arbitrary subset of the real line consisting of an even number of points. Mark the elements of $P$ on the real line. We shall let $P(0)$ be the left half of $P$ and $P(1)$ be the right half of $P$. In general, if $P\left(i_{1}, i_{2}, \ldots, i_{k}\right)$ is a subset of $P$ consisting of an even number of elements, where each $i_{j}$ is an integer modulo 2 , we shall let $P\left(i_{1}, i_{2}, \ldots, i_{k}, 0\right)$ be the subset which consists of the left half of $P\left(i_{1}, i_{2}, \ldots, i_{k}\right)$ and $P\left(i_{1}, i_{2}, \ldots, i_{k}, 1\right)$ the subset consisting of the right half of $P\left(i_{1}, i_{2}, \ldots, i_{k}\right)$.

Definition 2.3. Let $P$ be a periodic orbit of order $m \cdot 2^{r}$ for a function $f \in C^{0}(I, I)$, where $m$ and $r$ are some positive integers and $m$ is odd. The orbit $P$ is said to satisfy Block's condition for simple orbits if for each positive integer $k \leqslant r$ and for each sequence $i_{1}, i_{2}, \ldots, i_{k}$ of integers modulo $2, f^{2^{k-1}}\left(P\left(i_{1}, i_{2}, \ldots, i_{k}\right)\right)=$ $P\left(i_{1}, i_{2}, \ldots, i_{k}+1\right)$.

$P$ is said to violate Block's condition at the nth stage for some positive integer $n \leqslant r$ if there exists a sequence $i_{1}, i_{2}, \ldots, i_{n}$ of integers modulo 2 such that $f^{2^{n-1}}\left(P\left(i_{1}, i_{2}, \ldots, i_{n}\right)\right) \neq P\left(i_{1}, i_{2}, \ldots, i_{n}+1\right)$, but for every positive integer $k<n$, and every sequence $i_{1}, i_{2}, \ldots, i_{k}$ of integers modulo $2, f^{2^{k-1}}\left(P\left(i_{1}, i_{2}, \ldots, i_{k}\right)\right)=$ $P\left(i_{1}, i_{2}, \ldots, i_{k}+1\right)$.

III. Proof of Theorem II. The proof of our Theorem II is based on the following proposition.

Proposition 3.1. Consider any $f \in C^{0}(I, I)$. If $f$ has a minimum periodic orbit of an odd period $m$, then for each positive integer $n, f$ has a periodic orbit of order $2(m+n)$, which violates Block's condition at the first stage.

Proof. Suppose $f$ has a minimum periodic orbit $P$ of an odd order $m$ in the interval $I$. By Proposition 2.2, the orbit $P$ must be located in the interval $I$ either in the order of

$$
p_{m-1}<p_{m-3}<\cdots<p_{2}<p_{1}<p_{3}<\cdots<p_{m-2}<p_{m}
$$

or its "reflection" on the real line, where for each $i$ with $1<i<m-1, f\left(p_{i}\right)=p_{i+1}$ and $f\left(p_{m}\right)=p_{1}$. In any case, since $f\left(\left[p_{2}, p_{1}\right]\right) \supset\left[p_{2}, p_{3}\right]$, there must be a point $p_{0}$ in the interval $\left[p_{2}, p_{1}\right]$ such that $f\left(p_{0}\right)=p_{1}$. Without loss of generality, we may assume that

$$
p_{m-1}<p_{m-3}<\cdots<p_{2}<p_{0}<p_{1}<p_{3}<\cdots<p_{m-2}<p_{m} .
$$

Let $I_{1}=\left[p_{0}, p_{1}\right], I_{2}=\left[p_{2}, p_{0}\right], I_{3}=\left[p_{1}, p_{3}\right]$ and, in general, $I_{i}=\left[p_{i-2}, p_{i}\right]$ or $\left[p_{i}, p_{i-2}\right]$ for each $i=3,4, \ldots, m$. From the assumption on the points $p_{i}$, the 
associated digraph of these intervals must contain a cycle $I_{m} I_{1} I_{2} \cdots I_{m-1}$. Also since $f\left(p_{m-2}\right)=p_{m-1}$ and $f\left(p_{m}\right)=p_{1}$, there is a directed arc from $I_{m}$ to $I_{m-1}$. Let $C_{1}$ be the cycle $I_{m} I_{1} I_{2} \cdots I_{m-1}$ of length $m$ and $C_{2}$ be the cycle $I_{m} I_{m-1}$ of length 2 . Now, let any integer $n$ be given. The digraph contains a cycle $K$ of length $2(m+n)$ obtained by tracing the cycle $C_{1}$ twice, and then, tracing the cycle $C_{2} n$ times. By Proposition 2.1, there must exist a point $q \in I_{m}$ such that $f^{i}(q)$ enters each of the successive intervals of the cycle $K$ as $i$ increases from 1 to $2(m+n)-1$, and $f^{2(m+n)}(q)=q$. Note that the point $f^{i}(q)$ has to enter the interiors of the successive intervals of the cycle $K$. This is because the endpoints of these intervals, except for $p_{0}$, are iterates of $p_{1}$. If any of the iterates $f^{i}(q)$ becomes an endpoint of any of these intervals, then since $f^{2(m+n)}(q)=q, q$ itself must be an iterate of $p_{1}$, and therefore, the iterates of $q$ cannot go through the intervals of $K$ in the given order. Also note that the cycle $K$ is not composed of a single shorter cycle repeated several times. Consequently, the point $q$ is not of a period shorter than $2(m+n)$ for the function $f$.

We still need to show that the orbit $Q$ of $q$ under $f$ violates Block's condition at the first stage. Let

$$
U(f)=\{x \in I: f(x)>x\} \quad \text { and } \quad D(f)=\{x \in I: f(x)<x\} .
$$

Note that for Block's condition to be satisfied at the first stage, $Q(0)$, the left half of $Q$, must be a subset of $U(f)$, and $Q(1)$, the right half of $Q$, must be a subset of $D(f)$. In particular, the sets $Q \cap U(f)$ and $Q \cap D(f)$ must have exactly the same number of points.

On the other hand, since $f^{i}(q)$ belongs to the interiors of the successive intervals of the cycle $K$, it is easy to see that intervals $I_{m-1}$ and $I_{m}$ each contains $2+n$ points of $Q$, and each of the other intervals $I_{i}$ for $i=1,2, \ldots, m-2$ contains exactly two points of $Q$. From the way that the intervals $I_{1}, I_{2}, \ldots, I_{m}$ are located on the real line, we conclude that

$$
\begin{gathered}
Q \cap I_{i} \subset U(f) \text { for each even integer } i \text {, and } \\
Q \cap I_{i} \subset D(f) \text { for each odd integer } i \text {, where } 1 \leqslant i \leqslant m .
\end{gathered}
$$

But $m$ is an odd integer. There are more intervals $I_{i}$ with $i$ odd than there are $I_{i}$ 's with $i$ even. Consequently, there are more points in $Q \cap D(f)$ than there are points in $Q \cap U(f)$. This contradicts the conclusion of the preceding paragraph, and hence, the orbit $Q$ must violate Block's condition at the first stage.

To prove our Theorem II, we need one more lemma.

LEMMA 3.2. Let a function $f \in C^{0}(I, I)$ be given. If $g=f^{2^{k}}$ has a periodic point $x_{0}$ of period $m \cdot 2^{t}$ for some positive integer $t$ and some positive odd integer $m$, then $x_{0}$ is a periodic point of period $m \cdot 2^{t+k}$ for the function $f$. Furthermore, if the orbit of $x_{0}$ under $g$ violates Block's condition for simple orbits, so does the orbit of $x_{0}$ under $f$.

Proof. Since $f^{m \cdot 2^{t+k}}\left(x_{0}\right)=g^{m \cdot 2^{t}}\left(x_{0}\right)=x_{0}$, the point $x_{0}$ is a periodic point for $f$. Let $h$ be the period of $x_{0}$ for $f$. Then $h$ divides $m \cdot 2^{t+k}$. Also since $g^{h}\left(x_{0}\right)=x_{0}, h$ is a multiple of $m \cdot 2^{t}$. Therefore, $h=m \cdot 2^{r}$ for some integer $r$ such that $t \leqslant r \leqslant t+k$. We contend that $r=t+k$, for otherwise, $r \leqslant k+(t-1)$. Choose $s \geqslant 0$ such that 
$k+(t-1)=r+s$. Then

$$
\begin{aligned}
& g^{m \cdot 2^{t-1}}\left(x_{0}\right)=f^{m \cdot 2^{k+1-1}}\left(x_{0}\right)=f^{m \cdot 2^{r+1}}\left(x_{0}\right) \\
& =f^{m \cdot 2^{r}} \circ f^{m \cdot 2^{r}} \circ \cdots \circ f^{m \cdot 2^{r}}\left(x_{0}\right) \quad\left(2^{s} \text { times }\right) \\
& =x_{0} \text {. }
\end{aligned}
$$

This contradicts the assumption that $m \cdot 2^{t}$ is the least positive integer such that $g^{m \cdot 2^{t}}\left(x_{0}\right)=x_{0}$. Hence, $r=t+k$ and $h=m \cdot 2^{t+k}$.

The assertion that if the orbit of $x_{0}$ under $g$ violates Block's condition, so does that of $x_{0}$ under $f$ follows directly from the definition.

REMARK. One may easily construct an example to show that the assertion of the preceding lemma is no longer true if the integer $t=0$.

Proof of Theorem II. Let $p$ be a periodic point of period $m \cdot 2^{k}$ for some function $f \in C^{0}(I, I)$, where $k$ is a nonnegative integer and $m$ an odd integer $>1$. Note that if we can prove Theorem II for two other such integers $k^{\prime}$ and $m^{\prime}$ such that either $k^{\prime}=k$ but $m^{\prime}<m$, or $k^{\prime}<k$ and $m^{\prime}$ is an arbitrary odd integer $>1$, then the assertion would also be true for the given values of $k$ and $m$. Thus, without loss of generality, we may assume that $p$ is a minimum periodic point of $f$. Then, $p$ is a minimum periodic point of order $m$ for the function $f^{2^{k}}$. By Proposition 3.1, for each positive integer $n, f^{2^{k}}$ has a periodic point $q$ of period $2(m+n)$, whose orbit violates Block's condition. Then by Lemma 3.2, $q$ is a periodic point of period $(m+n) \cdot 2^{k+1}$ for the function $f$ such that the orbit of $q$ violates Block's condition. This proves the first case of Theorem II.

For case (2) of Theorem II, since $f$ has a periodic point of period $m \cdot 2^{k}$, by Sharkovskii's theorem, $f$ also has a periodic point $p_{0}$ of period $3 \cdot 2^{k+1}$. The point $p_{0}$ is therefore a periodic point of period 3 for the function $f^{2^{k+1}}$. Since a point of period 3 is always a minimum periodic point, we may apply Proposition 3.1 with $m=3$ to the point $p_{0}$ and the function $f^{2^{k+1}}$ to get a periodic point $q$ of period $(3+n) \cdot 2$, where $n$ is an arbitrary positive integer and the orbit of $q$ under $f^{2^{k+1}}$ violates Block's condition. Then by Lemma 3.2 again, $q$ is a periodic point of period $(3+n) \cdot 2^{k+2}$ for $f$ such that the orbit of $q$ violates Block's condition.

Finally, consider the case (3) of Theorem II. Let any positive integers $r$ and $s$ be given such that $r>k+2$. The assertion for case (3) follows immediately if we substitute the number $s \cdot 2^{r-(k+2)}-3$ for the integer $n$ in the conclusion of case (2). This finishes the proof of Theorem II.

ACKNOWLEDGEMENT. The author wishes to thank both the referee and Professor Marilynn L. Livingston for detecting and correcting an error contained in the original proof of Proposition 3.1.

\section{REFERENCES}

1. L. Block, Simple periodic orbits of mappings of the interval, Trans. Amer. Math. Soc. 254 (1979), 391-398.

2. L. Block, J. Guckenheimer, M. Misiurewicz and L. Young, Periodic points and topological entropy of one dimensional maps (Proc. Conf. Global Theory of Dynamical Systems), Lecture Notes in Math., vol. 819, Springer-Verlag, Berlin, 1980, pp. 18-34.

3. C.-w. Ho, On the structure of the minimum orbits of periodic points for maps of the real line (to appear). 
4. C.-w. Ho and C. Morris, A graph theoretic proof of Sharkovsky's theorem on the periodic points of continuous functions, Pacific J. Math. 96 (1981), 361-370.

5. Z. Nitecki, Topological dynamics on the interval, Ergodic Theory and Dynamical Systems, Vol. II, Progress in Math., Birkhäuser, Boston, Mass., 1981.

6. A. N. Sharkovskii, Co-existence of the cycles of a continuous mapping of the line into itself, Ukranian Math. Z. 16 (1964), 61-71.

7. P. Stefan, $A$ theorem of Šarkovskii on the existence of periodic orbits of continuous endomorphisms of the real line, Comm. Math. Phys. 54 (1977), 237-248.

8. P. D. Straffin, Jr., Periodic points of continuous functions, Math. Mag. 51 (1978), 99-105.

DePartment of MAThematics, Southern Illinois University, EdWARDSVille, Illinois 62026 